\title{
A Study of IgA Antibody Response to Different Mycobacterium tuberculosis Antigens in the Diagnosis and Monitoring of Pulmonary Tuberculosis
}

\author{
Janaina Miranda Bezerra ${ }^{1}$, Sandra Trevisan Beck ${ }^{2}$, Kelly Aparecida Kanunfre ${ }^{4}$, Olavo Munhoz Leite ${ }^{3}$ and Antônio Walter Ferreira ${ }^{4,5}$ \\ ${ }^{1}$ Federal University of Maranhão, Maranhão, MA; ${ }^{2}$ Federal University of Santa Maria, Rio Grande do Sul, RS; ${ }^{3}$ Hospital das Clinicas, \\ University of São Paulo; ${ }^{4}$ São Paulo Institute of Tropical Medicine; ${ }^{5}$ Department of Infectious Diseases, Faculty of Medicine, University of São \\ Paulo; São Paulo, SP, Brazil
}

\begin{abstract}
We evaluated the performance of the ELISA technique in the detection of IgA antibodies against different Mycobacterium tuberculosis antigenic preparations in serum samples from 49 patients with pulmonary tuberculosis collected before and after the start of specific treatment. The controls consisted of serum samples from healthy patients without any prior contact with the bacteria and serum samples from patients with other pneumopathies. Glycolipid antigen gave the best diagnostic performance, with a sensitivity of $88 \%$ and specificities varying from 88 to $100 \%$ in the control groups. These antigens constitute a powerful tool for the diagnosis and monitoring of patients with pulmonary tuberculosis.
\end{abstract}

Key-Words: Tuberculosis, IgA, serological diagnosis, ELISA, glycolipid antigen.

There are many different mechanisms involved in immunological defense against Mycobacterium tuberculosis. The result of the interaction of these mechanisms and bacillus virulence factors determines whether acute or asymptomatic latent infection is established. Among the risk factors for individuals in the latent phase to develop the disease are infection by the human immunodeficiency virus (HIV), malnutrition, diabetes mellitus and the use of immunosuppressive drugs. Conditions such as immunological deficiencies of a genetic nature or genetic conditions related to the HLA system, for example, also lead to greater susceptibility to development of active tuberculosis [1].

During airway contamination, the bacilli can adhere to and invade the mucosa in the respiratory tract [2]. Depending on the individual's characteristics and the particular antigen involved in the stimulation, cellular or humoral responses may be activated. Although the role of secretory IgA in protecting mucosal surfaces is widely accepted, the role of serum IgA has been little investigated. This immunoglobulin may be involved in removing antigens from the circulatory system and transferring them to the bile [3]. In addition, it has been shown that this antibody has the ability to opsonize and agglutinate the antigen and to fix complement [4]. IgA response is particularly interesting, as this immunoglobulin has been shown to be produced in large quantities in patients with active pulmonary disease.

Control of tuberculosis depends mainly on the rapid detection and treatment of active cases. Given that $90 \%$ of the clinical forms are pulmonary and that transmission takes place via the airways, early diagnosis is an important means of controlling the disease.

Received on 20 July 2008; revised 11 December 2008.

Address for correspondence: Dr. Janaina M. Bezerra. Rua Urbano Santos s/n Centro. Zip code: 65900-000 Imperatriz-MA. Phone: (99) 35246200. E-mail: mbjanaina@hotmail.com.

The Brazilian Journal of Infectious Diseases

2009;13(1):53-58. (C) 2009 by The Brazilian Journal of Infectious Diseases and Contexto Publishing. All rights reserved.
Currently, diagnosis is based on clinical findings and laboratory tests. Traditional microbiological diagnostic methods have a number of limitations when used to detect microorganisms, particularly in patients with paucibacillary tuberculosis or extrapulmonary forms of the disease. Furthermore, because of the high prevalence of nontuberculous mycobacteria, the long time required for bacterial growth and species identification using biochemical tests in material from coinfected patients makes these tests difficult to perform [5-7].

The purified protein derivative (PPD) used in intradermal tuberculin tests to detect delayed-type hypersensitivity (the Mantoux test) has limited diagnostic value, as clinically healthy individuals can have a positive tuberculin test (PPD) $(>10 \mathrm{~mm})$, without this reactivity being reflected in tests for specific antibodies [8-10]. Furthermore, people who have been vaccinated with bacillus Calmette-Guérin (BCG) and/or have been in contact with other species of mycobacteria can have positive results as well [11].

Molecular methods for detecting the structural components of the bacteria have limitations related to cost and reproducibility, which makes their use on a large scale difficult. Serological tests based on the detection of antibodies are promising because they do not depend on the detection of mycobacteria, are both quick and sensitive, are easy to perform and are cheaper than tests based on the amplification of nucleic acids. In addition, they allow diagnosis of paucibacillary and extrapulmonary tuberculosis and can be used to detect the active phase of the disease. In this context, the choice of antigen is important, and all the characteristics of the response kinetics must be assessed to ensure the best interpretation of the results.

Given the limitations of traditional methods and the need for a better understanding of the response of IgA in human pulmonary tuberculosis, the objective of this study was to investigate whether this class of immunoglobulin could be used as a marker for the acute form of the disease and to identify variations in the level of such immunoglobulins during the clinical evolution of patients receiving therapy. The 
following three mycobacterial antigens were used in our study: glycolipid, recombinant protein, and crude antigen obtained, by sonication, from a culture of M. tuberculosis.

\section{Material and Methods}

Study Population

The sera samples used in this study were collected from June 2000 to April 2002. The study was approved by the local ethical committee of the Hospital das Clinicas de São Paulo. Written informed consent was obtained from each participant.

\section{Group I - Patients with Tuberculosis}

Fifty-two patients with clinical symptoms suggestive of tuberculosis, with an average age of 36 years (minimum 14 and maximum 75 years), who had been seen in the outpatient unit at the Hospital das Clínicas, São Paulo, SP, and had had their diagnosis confirmed by direct sputum-smear microscopy and/or culture and the species identified by microbiological or molecular analysis were included. When direct sputum examination was not possible, cytology of bronchial alveolar lavage, lung biopsy or lymph node puncture was performed. All the patients had negative HIV serology.

Forty-eight serum samples were collected from patients with pulmonary tuberculosis $(n=44)$ and tuberculous lymphadenitis $(n=4)$ before the treatment started. Serological follow-up was carried out by collecting blood samples at two distinct times, namely, after two months of treatment and at the end of the treatment (after six months), as advocated by WHO. Serum samples were collected from 42 patients with pulmonary tuberculosis and from four with tuberculous lymphadenitis.

\section{Group II - Control Group}

We analyzed serum samples from 20 individuals considered healthy who declared that they had not had any contact with patients with pulmonary tuberculosis, whether relatives or not, and did not have any clinical symptoms of tuberculosis at the time they were included in our study. Tuberculin tests (PPD) were not carried out in these individuals.

\section{Group III - Patients with Other Pneumopathies $(N=49)$}

Serum samples from patients with other pulmonary diseases for whom the possibility of pulmonary tuberculosis had been excluded were collected in the pneumology outpatient unit at the Hospital das Clínicas, São Paulo.

\section{Group IV - Contacts $(N=25)$}

Nine serum samples were collected from individuals who had been exposed to tubercular patients during the course of their work and 16 samples from individuals who had come into contact with patients with active tuberculosis. Although tuberculin tests (PPD) were requested, these could not be carried out in all the individuals in this group. Patients had difficulty adhering to the study because they had to return to the outpatient unit for the skin tests to be carried out and subsequently read (72 hours). Consequently, this parameter was not taken into account in the analysis.

\section{M. tuberculosis antigens}

Whole-protein antigens were obtained after culturing a clinical strain of $M$. tuberculosis isolated in 2000 and characterized by microbiological and molecular methods, as described by Beck in 2005. The recombinant antigen combination TbF6/DPEP was supplied by Corixa Corp., Seattle, WA, USA.

Glycolipid antigen, purified according to WATANABE et al, 1995, on Nunc PolySorp plastic plates, was supplied by the R\&D department at bioMérieux SA exclusively for use in this study.

\section{ELISA(Enzyme -Linked Immunosorbent Assay)}

Flat-bottomed polystyrene plates (Nunc-Immuno Module Maxisorp F 16, DENMARK) were sensitized with the wholeprotein antigen or recombinant antigen using $100 \mu \mathrm{L} /$ well at the appropriate concentrations for each antigen (whole-protein antigen at a concentration of $0.5 \mu \mathrm{g} / \mathrm{well}$ and recombinant antigen at a concentration of $400 \mathrm{ng} /$ well), diluted in a $0.06 \mathrm{M}$ $\mathrm{pH} 9.6$ carbonate/bicarbonate buffer and incubated for two hours at $37^{\circ} \mathrm{C}$ and again for 18 hours at $4^{\circ} \mathrm{C}$. The antigen solution was removed and the wells blocked for two hours at $37^{\circ} \mathrm{C}$ with buffer phosphate saline (PBS) containing 5\% skim milk. The plates were then washed with PBS-T (PBS containing $0.05 \%$ Tween 20 ), followed by two washes with distilled water. The plates were placed in an incubator at $37^{\circ} \mathrm{C}$ for 4 hours, wrapped individually in aluminum foil, sealed and kept at $4^{\circ} \mathrm{C}$ until use.

To carry out the test with the whole-protein antigen and recombinant antigen, the sensitized plates were incubated with $100 \mu \mathrm{L}$ of the sera diluted $1 / 20$ in PBS containing $2 \%$ skim milk. After incubation for 30 minutes at $37^{\circ} \mathrm{C}$ in a moist chamber, the sera were removed and the plates washed five times with PBS-T. The anti-IgA enzymatic conjugate diluted 1/7,500 in the same diluent as the serum was then added $(100 \mu \mathrm{L} /$ cavity). After incubation at $37^{\circ} \mathrm{C}$ for 30 minutes in a moist chamber and a new cycle of washes, $100 \mu \mathrm{L} /$ well of the chromogenic substrate tetramethylbenzidine/hydrogen peroxide (TMB/ $\mathrm{H}_{2} \mathrm{O}_{2}$ ) was added and the plate was incubated for an additional 30 minutes at $37^{\circ} \mathrm{C}$ in a moist chamber kept in the dark. The enzymatic reaction was stopped by adding $50 \mu \mathrm{L}$ of $2 \mathrm{~N}$ sulphuric acid, and the plates read at $450 \mathrm{~nm}$ in a Titertek microplate reader.

To carry out the test with glycolipid antigen, the plates were incubated with $100 \mu \mathrm{L}$ of the sera diluted $1 / 250$ in PBS containing $2 \% \mathrm{BSA}$. After incubation for 30 minutes at $37^{\circ} \mathrm{C}$ in a moist chamber, the sera were removed by quickly turning the plates upside down and washing them six times with PBS$\mathrm{T}$ and three times with bidistilled water. The anti-IgA enzyme conjugate, diluted 1/7,500 in the supplied diluent, was then added $(100 \mu \mathrm{L} /$ well $)$. After incubation at $37^{\circ} \mathrm{C}$ for one hour in a moist chamber, followed by a new cycle of washes, $100 \mu \mathrm{L} /$ 
well of the chromogenic solution O-phenylenediamine dihydrochloride with hydrogen peroxide $\left(\mathrm{OPD} / \mathrm{H}_{2} \mathrm{O}_{2}\right)$ was added and the plate was incubated for 30 minutes at ambient temperature in the dark. The enzymatic reaction was stopped by adding $50 \mu \mathrm{L}$ of $2 \mathrm{~N}$ sulphuric acid, and the plates were read at $492 \mathrm{~nm}$ in a Titertek microplate reader.

The threshold for the test was obtained using the samples from patients with tuberculosis in pretreatment $(\mathrm{n}=48)$ and from healthy patients $(n=20)$. The cutoff was set at two standard deviations above the mean of the optical densities (O.D.) for sera from healthy individuals based on analysis of the ROC (Receiver Operating Characteristic) curve using Win Episcope 2.0 software.

\section{Statistical Analysis}

The results were expressed as percentages when appropriate. The optical densities were calculated and a $\mathrm{p}$ value $<0.05$ was considered statistically significant for all tests.

\section{Results}

To define the reactivity threshold more accurately, ROC curves were drawn, taking into account the cutoff points established with one and two standard deviations and the interval between them, to find a value that would improve the test's specificity without a significant loss of sensitivity. It can be seen in Figure 1 that the glycolipid antigen had the largest area under the curve (99.4\%), with a sensitivity of $88 \%$ and a specificity of $100 \%$.

After the reactivity threshold had been determined for the different antigens, using sera from healthy individuals, the ELISA test was carried out on sera from patients with other pneumopathies and from contacts.

A 2 x 2 contingency table was used to calculate Youden's $\mathrm{J}$ index, which evaluates the diagnostic efficiency of the tests used. The glycolipid antigen had the highest J index, followed by TBF6/DPEP and the whole-protein antigens.

The glycolipid and recombinant (TBF6/DPEP) antigens had the best specificity values when the serum samples from healthy individuals were studied (100 and 90\%, respectively). There was a noticeable drop in the specificity of the recombinant proteins, however, in the group that included other pneumopathies $(74 \%)$ and in the contacts $(88 \%$, Table 1$)$.

The glycolipid antigen (PGL-Tb1) had the smallest variation in specificity among the groups analyzed; the specificity for healthy individuals was $100 \%, 88 \%$ for patients with other pneumopathies, and $80 \%$ for individuals who had had contact with patients with active tuberculosis, corresponding to an overall specificity of $89 \%$.

Serological follow-up to determine IgA reactivity to $M$. tuberculosis was conducted in 50/52 patients. The median optical densities (MdOD) for the total protein antigen were similar at each of the three serological follow-ups (Figure 2, $\mathrm{p}>0.05$, Kruskal-Wallis method); the MdOD for the TBF6/DPEP and glycolipid (PGL-Tb1) antigens were different at each of the three follow-ups. The MdOD for ELISA with glycolipid antigen were found to differ significantly between the first, second and third serum samples ( $p<0.0062$, Dunn's method). Figure 3 shows the median absorbances during serological follow-up of the patients using glycolipid antigen. The drop in reactivity between the first samples (pretreatment), the samples at two months and the samples at the end of the treatment (six months on average) was significant $(\mathrm{p}=$ 0.00000466 , Friedman repeated-measures method).

Of the 44 patients with active pulmonary tuberculosis, 35 were sputum-smear positive, eight were negative, and for one patient the result of the sputum smear test was not available. The glycolipid antigen had the best performance; it detected $91 \%$ of the cases of pulmonary tuberculosis (40/44), made up of 33 of the 35 patients with positive sputum smears and seven of the eight with negative sputum smears.

Figure 4 shows the median optical densities for serum samples from patients with pulmonary tuberculosis and positive sputum smears and patients with negative sputum smears. Significant differences were found when wholeprotein and glycolipid antigen was used ( $\mathrm{p}=0.012$ and $\mathrm{p}=0.034$, respectively, non-parametric Mann-Whitney test).

\section{Discussion}

Studies have shown that $\operatorname{IgG}_{1}$ and $\operatorname{Ig} A$ antibodies are formed in response to various mycobacterial products, including components of the cell wall. This underlines the need to examine the role of humoral immunity in mycobacterial infections in greater detail $[9,14,15]$.

We evaluated the performance of ELISA tests using the following three antigens: recombinant antigen combination (TBF6/DPEP), glycolipid antigen and whole-protein antigen produced from soluble proteins obtained after sonication of a clinical strain of M. tuberculosis. After protocols had been defined, we used standardized tests with serum samples from healthy individuals $(\mathrm{n}=20)$ to determine the cutoff for the tests. As in other studies [10], the ROC curve was used to determine the best specificity values for the tests without a major loss in sensitivity and to identify the most accurate test (Figure 1).

Julian et al. (2002) showed that adult tuberculosis patients have a specific IgA response to glycolipid antigens. However, they found that tests detecting immunoglobulin A had lower sensitivity (66\%) and greater specificity (88\%) than IgG. The sensitivity and specificity found in our study were 88 and $89 \%$, respectively.

While not considered ideal for use in commercial tests, the sensitivity of the IgA ELISA was within the range recommended by $\mathrm{WHO}$ (greater than $80 \%$ when the reference is IgG antibody detection). However, the six sera from tuberculosis patients that were not considered to be reactive by the IgA ELISA with glycolipid antigen had optical density values close to the reactivity threshold (20\%).

A number of factors can influence the reactivity of the test, such as the patient's immunological "status" and the stage and duration of the disease at the moment that the 
Table 1. ELISA parameters for IgA antibodies anti-Mycobacterium tuberculosis using three different antigens.

\begin{tabular}{|c|c|c|c|c|c|c|c|}
\hline Antigens & Cut-off & $\mathrm{S}(\%)$ & Population & Sp (\%) & PPV (\%) & NPV (\%) & $\mathbf{J}$ index \\
\hline \multirow{3}{*}{ Whole-protein: } & 0.370 & 69 & Healthy & 75 & 88 & 48 & \\
\hline & & & Contacts & 72 & 78 & 55 & 0.40 \\
\hline & & & $\mathrm{OP}$ & 55 & 62 & 63 & \\
\hline \multirow[t]{3}{*}{ TBF6/DPEP } & 0.450 & 58 & Healthy & 90 & 94 & 45 & \\
\hline & & & Contacts & 88 & 91 & 66 & 0.48 \\
\hline & & & $\mathrm{OP}$ & 74 & 71 & 63 & \\
\hline \multirow[t]{3}{*}{ Glycolipid } & 0.137 & 88 & Healthy & 100 & 100 & 77 & \\
\hline & & & Contacts & 80 & 92 & 75 & 0.88 \\
\hline & & & $\mathrm{OP}$ & 88 & 87 & 87 & \\
\hline
\end{tabular}

$\mathrm{S}=$ sensitivity; $\mathrm{Sp}=$ specificity; $\mathrm{PPV}=$ positive predictive value, $\mathrm{NPV}=$ negative predictive value; $\mathrm{OP}=$ other pneumopathies; $\mathrm{J}$ index $=$ calculated with the general specificity value.

Figure 1. Evaluation of three antigens using ELISA by Receiver Operating Characteristic (ROC) Curves.

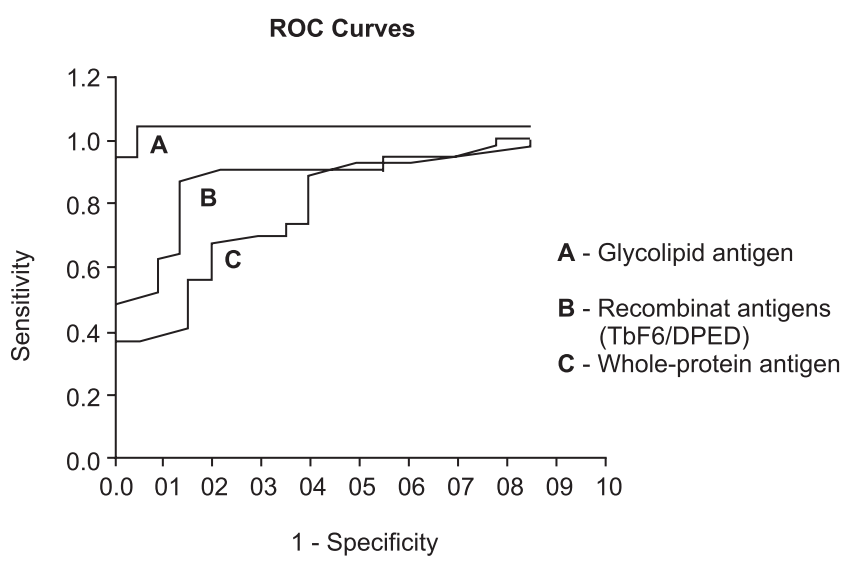

Figure 2. Median optical densities (MdOD) observed in the analysis of sera from 50 patients at the different serological follow-ups for different antigens. MdOD $1^{\text {st }}$ sample $=$ MdOD of the samples collected before therapy had started; MdOD 2 nd sample $=$ MdOD of the samples collected during the $2^{\text {nd }}$ month of treatment; MdOD $3^{\text {rd }}$ sample $=$ MdOD of the samples collected at the end of treatment.

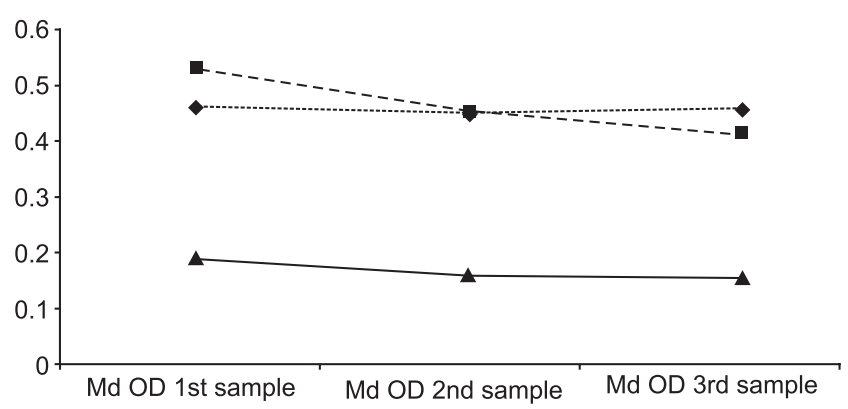

$\rightarrow--$ Whole-protein $\quad \rightarrow-$ TbF6/DPEP $\rightarrow$ PGL-Tb1
Figure 3. Median OD values for IgA ELISA using glycolipid antigen during serological follow-up of patients with active tuberculosis. $\mathrm{T} 0=$ samples collected before treatment; $\mathrm{T} 2=$ samples collected during the $2^{\text {nd }}$ month of treatment; T6 = samples collected at the end of treatment.

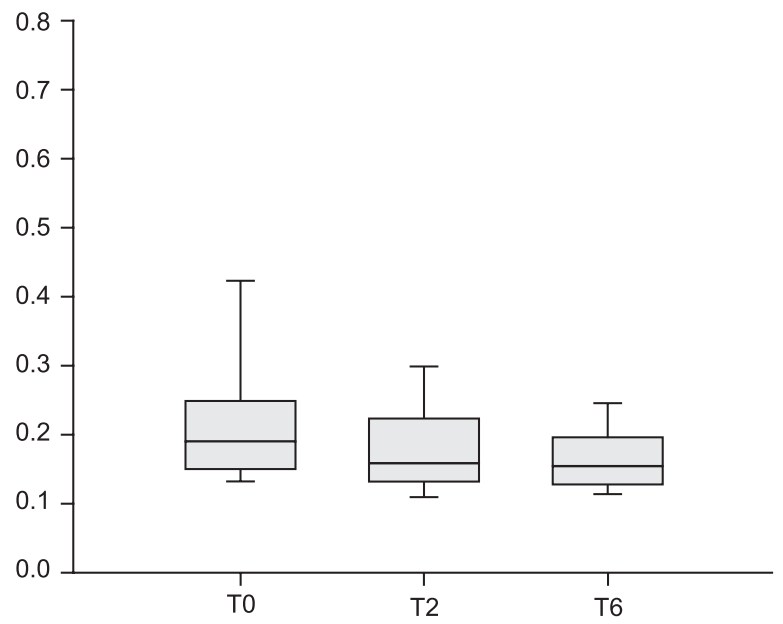

patient was included in the study [16-18]. When the ELISA tests using different antigens were carried out with sera from groups III and IV, there was less variation in specificity with the glycolipid antigen (88\%-100\%). In studies where there is a significant number of samples from groups III and IV, variations in specificity as a result of deregulation of the immune system are often found [18-20].

Studies carried out by BECK (2005) to detect IgG antibodies against a recombinant antigen combination (TBF6/DPEP) revealed sensitivity and general specificity values of $85 \%$ and $91 \%$, respectively. In our study, we also found that the IgA response against this antigen combination had a noticeably higher specificity for the healthy individuals than for the contacts and individuals with other pneumopathies; it varied from $74 \%$ to $90 \%$. The false-positive reactions in the group of individuals with other pneumopathies can also be attributed to the presence of hypergammaglobulinemia, which is common in nontuberculous pneumonias [21]. 
Figure 4. Median ODs observed in the ELISAcompared with the results of sputum-smear microscopy. A p value $<0.05$ was considered significant (Mann - Whitney test).
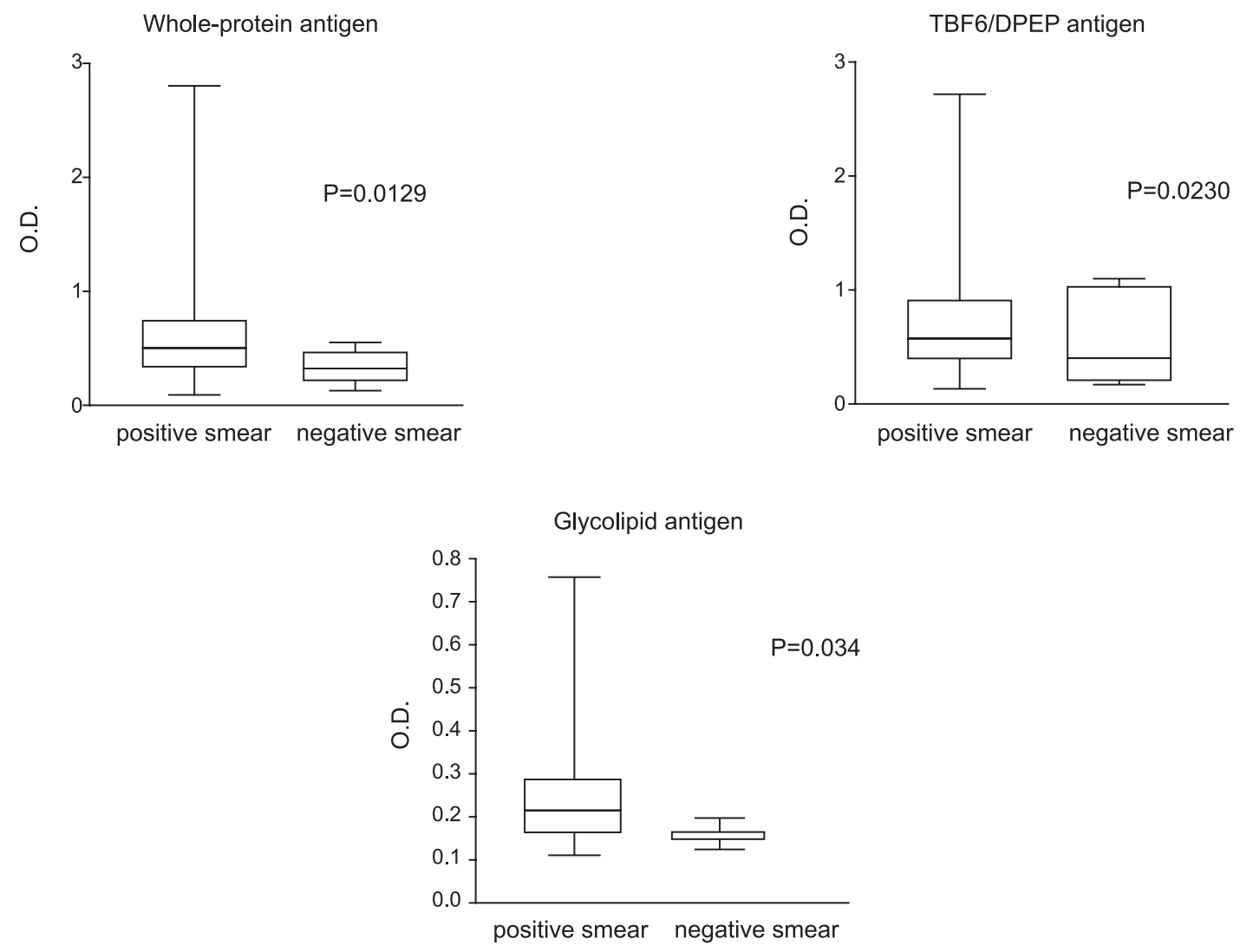

The whole-protein antigen had a lower specificity value, and there was greater variation in this parameter among the control groups. This can be explained by the many antibodies that can be produced for the different species of mycobacteria that share epitopes with M. tuberculosis. Although the population in Brazil is vaccinated with an attenuated strain of bacillus Calmette-Guérin (BCG), the antibodies produced by immunization do not appear to interfere in the reactivity when sera from vaccinated individuals are tested by ELISA [10,2224] and are not involved in the positive reactions found in the control group in our study.

Youden's index showed that the glycolipid antigen had the best diagnostic efficiency. However, as the mycobacterial antigens that were analyzed have different molecular structures, there was no correlation between the serological results for the different tests.

Various studies report the presence of IgA antibodies against $M$. tuberculosis as a marker of recent infection, while others refer to extensive, active infection and miliary tuberculosis $[9,17,25]$. The former hypothesis appears to be related to the fact that this immunoglobulin is secreted during initial contact with the bacillus on mucosal surfaces (secretory $\operatorname{IgA}$ ) and that mycobacterial antigens (such as glycolipids) interact with specific receptors in the host-defense cells, stimulating the release of cytokines (IL-4, 6 and 10) that have adjuvant effects, such as inducing immunoglobulin isotype switching (IgM to IgA) [26]. However, inour study IgA antibodies against the glycolipid antigen were detected irrespective of the time of onset of the symptoms ( 0 to 24 months), suggesting that the IgA response is present in both recently acquired infections and those reported more than two years previously and is thus more associated with active disease.

Although the stage and duration of infection are important factors in determining the presence of the immunoglobulin, other factors, such as the formation of immune complexes, the characteristics of the individual's immune system and genetic factors, particularly those related to the HLA system, can also interfere with the humoral response against glycolipid antigen.

Although the number of cases of pulmonary tuberculosis patients with a negative smear test was small in our study, we found that the glycolipid antigen had the best performance in the IgA ELISA. Alifano et al. (1997) studied IgA response against Kp-90 antigen in patients with negative smear tests and reported a sensitivity of approximately $68 \%$. As also found in other studies, most of the patients with negative sputum smears who were positive in the IgA ELISA in our study had pulmonary infiltrates without cavity lesions. 
The heterogeneity of the immune response can be explained by the different types of antigen released by the mycobacteria during the various stages of the disease. Cavity lesions have different stages of liquefaction and can vary in terms of the amount of bacilli in the cavity, bacillary replication and the metabolic changes that can take place [27].

After two and six months of therapy, a drop in serological reactivity for the ELISA IgA with glycolipid antigen (Figure 3) was observed in the serum samples from patients with tuberculosis. Most of the drugs used to treat tuberculosis act on the components of the cell wall. Glycolipids are present on the outermost surface of the mycobacteria and are therefore an easy target for therapeutic agents. Arikan et al. (1998) showed that anti-Kp90 IgA antibodies have a promising role in monitoring tuberculosis therapy.

The IgA ELISA had different results for the three antigens evaluated. The many different protein fractions present in the whole-protein antigen extract may explain its greater reactivity. We found that three patients were reactive for the crude and glycolipid antigen, making it important to analyze these patients more accurately, as despite the absence of clinical symptoms they might be developing latent tuberculosis.

Although IgA ELISA with glycolipid antigen gave the best results, we believe that the specificity of the test can be improved by using more specific glycolipid antigens that are only present in $M$. tuberculosis extract, by avoiding contamination during the antigen purification process and by preabsorption of the serum with mycobacteria other than $M$. tuberculosis. Our results suggest that IgA antibodies have potential clinical value for monitoring tuberculosis therapy, as $40 \%$ of the patients with tuberculosis in the study had a drop in reactivity during the follow-up period and $20 \%$ had negative serology at the end of the treatment.

\section{References}

1. Parrish N.M., Dick J.D., Bishai W.R. Mechanisms of latency in Mycobacterium tuberculosis. Trends in Microbiology 1998;6(3):106-12.

2. Middleton A.M., Chadwick M.V., Nicholson A.G., et al. Interaction of Mycobacterium tuberculosis with human respiratory mucosa. Tuberculosis 2002;82(2/3):69-78.

3. Russel M.W., Brown T.A., Mestecky J. Role os serum IgA. J Exp Méd 1981;153:968-76.

4. Porven G., Padin L., Müller V., et al. Accion de la immunoglobulina A secretoria sobre el Mycobacterium tuberculosis. Medicina (Buenos Aires) 1985;45:496-500.

5. Hopewell P.C. Impact of human immunodeficiency vírus infection on the epidemiology clinical features, management, and control of tuberculosis. Com Infect Dis 1992;15:540-7.

6. Dalcomo M.P., Kristki A.L. Tuberculosis co-infección por HIV. Rev Arg Torax 1993;54:29-54.

7. Barnes P.F., Bloch A.B., Davidson P.T., Snider D.E. Tuberculosis in patients with human immunodeficiency virus infection. N Engl J Med 1991;324:1644-50.

8. Beck S.T. Padronização e desenvolvimento de testes sorológicos para o diagnóstico da tuberculose humana. São Paulo, 168p. Tese de (Doutorado) - Faculdade de Ciências Farmacêuticas de São Paulo, Universidade de São Paulo, 2003.
9. Wilsher M.L., Hagan C., Prestidge R., et al. Human in vitro immune response to Mycobacterium tuberculosis. Tubercle and Lung Diseases 1999;79(6):371-7.

10. Alifano M., De Pasacalis R., Sofia M., et al. Evaluation of IgA mediated humoral immune response against the mycobacterial antigen P-90 in diagnosis of pulmonary tuberculosis. Chest 1997; 111:601-5.

11. Pottumarthy S., Morris A.J., Harrison A.C., Wells V.C. Evaluation of the tuberculin gamma interferon assay: potential to replace the Mantoux skin test. J Clin Microbiol 1999;37:3229-32.

12. Beck S.T., Leite O.M., Arruda R.S., Ferreira A.W. Combined Use of Western Blot/ELISA to Improve the Serological Diagnosis of Human Tuberculosis. Brazilian Journal of Infectious Diseases 2005;9(1):35-43.

13. Watanabe M., Honda I., Kawajiri K., et al. Distribution of antibody titres against phenolic glycolipids from Mycobacterium tuberculosis in the sera from tuberculosis patients and healthy controls. Res Microbiol 1995;146:791-7.

14. Schuler N.W., Rom W.N. The host immune response to tuberculosis. Am J. Respir Care Méd 1998;157:679-91.

15. Freedman A.G. Advances in antibody-mediated immunity against Mycobacterium tuberculosis: implications for a novel vaccine strategy. FEMS Immunol and Medical Microbiology 2003;39:9-16.

16. Julian E., Matas L., Perez A., et al. Serodiagnosis of tuberculosis: Comparison of Immunoglobulin A (IgA) response to Sulfolipid I with IgG and IgM response to 2,3 - Diacyltrehalose, 2,3,6 Triacyltrehalose and cord factor antigens. J Clin Microbiol 2002;40:3782-88.

17. Gupta S., Kumari S., Banwalikar J.N., et al. Diagnostic utility of the estimation of mycobacterial antigen A60 specific immunoglobulins IgM, IgA, IgG in the sera of cases of adult human tuberculosis. Tubercle Lung Dis 1995;76:418-24.

18. Julian E., Matas L., Hernandez A., et al. Evaluation of a new serodiagnosis tuberculosis test based on the immunoglobulin A detection against Kp-90 antigen. Int J Lung Tuberc Dis 2000; 4: 1082-85.

19. Chiang I.H., Suo J., Bai K.J., et al. Serodiagnosis of tubeculosis. A study comparasing three specific mycobacterial antigens. Am J Respir Care Med 1997;156:906-11.

20. Cocito C.G. Properties of the mycobacterial antigen complex A60 and its application to the diagnosis and prognosis of tuberculosis. Chest 1991;100:1687-93.

21. Bothamley G.H., Rudd R.M. Clinical evaluation of a serological assay using a monoclonal antibody (TBF72) to the $38 \mathrm{kDa}$ antigen of Mycobacterium tuberculosis. Eur Respir J 1994; 7:240-6.

22. Balestrino E.A., Daniel T.M., De Latini M.D.S., et al. Serodiagnosis of pulmonary tuberculosis in Argentina by enzyme-linked immunosorbent assay (ELISA) of $\mathrm{IgG}$ antibody to Mycobacterium tuberculosis. J Immunol 1992;148:1835-40.

23. Sada E., Brennan P.J., Herrar T, Torres M. Evaluation of lipoarabinomannan for the serological diagnosis of tubeculosis. J Clin Microbiol 1990;28(12):2587-90.

24. Zou Y.L., Zhang J.D., Chen M.H., et al. Serological analysis of pulmonary and extrapulmonary tuberculosis with enzyme-linked immunosorbent assay for anti A60 immunoglobulins. Clin Infect Dis 1994; 19:1084-91.

25. Arikan S., Tuncer S., Us D., et al. Anti-Kp 90 IgA antibodies in the diagnosis of active tuberculosis. Chest 1998; $114: 1253-7$.

26. Taha R.A., Kotsimbos T.C., Song Y.L., et al. IFN-gama and IL-12 are increased in active compared with inactive tuberculosis. Am J Respir Crit Care Med 1997;155(3):1135-9.

27. Mac Vandiviere H., Loring W.E., Melvin I., Willis S. The treated pulmonary lesion and its tubercle bacillus. II The death and resurrection. Am J Med Sci 1956:31-7. 